\title{
COVID-19: Weiter impfen und wachsam bleiben
}

\section{Impfangebot für EU-Bürger - Forschung an Virusvarianten}

\author{
Die COVID-19-Fallzahlen und auch die Zahl jener Patienten, die eine intensivmedizinische Behandlung \\ brauchen, sind im September wieder angestiegen: Experten sehen darin den Beginn einer vierten Welle. - \\ Noch ist die Pandemie nicht vorbei!
}

\section{Durchimpfungsrate}

Eineinhalb Jahre nach Beginn der COVID-19-Pandemie werden jede Sekunde 40 Menschen in Europa geimpft. „Die EU ist auf sehr gutem Weg, ihr gemeinsames Ziel zu erreichen: Bis Ende Juli haben mindestens $70 \%$ der Erwachsenen in der EU ein Impfangebot erhalten", berichtet Wolfgang Bogensberger, stellvertretender Leiter der Vertretung der Europäischen Kommission in Österreich. „Dass das so rasch möglich war, ist der beispiellosen Zusammenarbeit zwischen Forschung, Industrie, politischen Entscheidungsträgern und Gesundheitsbehörden zu verdanken“, ergänzt Renée GalloDaniel, Präsidentin des Verbandes der Impfstoffhersteller (ÖVIH).

\section{Impfdilemma vermeiden}

Gleichzeitig warnt sie auch: „Viele Impfungen werden mit der Zeit ein Opfer ihres eigenen Erfolges. Das bedeutet, dass die Krankheiten, gegen die sie schützen, irgendwann nur noch so selten auftreten, dass ein Gefühl der vermeintlichen Sicherheit entsteht. Die Impfbereitschaft geht dann oft zurück." Die Folge sei, dass die Krankheiten wieder stärker zurückkehren. „Diese Situation müssen wir bei COVID-19 unbedingt vermeiden", betont die Expertin. ob er oder sie sich impfen lassen oder wer vielleicht auf die zweite Teilimpfung verzichten wollte, sollte dies spätestens seit Auftreten der Delta-Variante nicht mehr tun", stellt Univ.-Prof. Dr. Karl Zwiauer vom Nationalen Impfgremium klar. „Alle, die nicht oder nur einfach geimpft sind, haben ein hohes Risiko sich mit dieser infektiöseren Variante anzustecken, die noch dazu auch häufiger zu schweren Verläufen führt. Daten aus Großbritannien zeigen eindeutig, dass eine Teilimpfung in diesem Fall nicht ausreichend ist. Vielen dürfte das noch nicht bewusst sein." Ein Verschieben der zweiten Teilimpfung über den empfohlenen Impfabstand hinaus - zum Beispiel wegen Urlaub - würde neben dem Risiko der eigenen Erkrankung auch zu einem höheren Risiko führen, Varianten nach Österreich einzuschleppen. Beim Impfen gehe es somit nicht nur um die Eigen-, sondern auch um die Fremdverantwortung.

Wegen der neuen Virus-Variante hat das Nationale Impfgremium (NIG) im Juni 2021 beschlossen, wieder zu jenen kürzeren Abständen zwischen den Teilimpfungen zurückzukehren, die ebenso in den Studien geprüft wurden und auch laut Zulassung empfohlen sind. Das sind 21 beziehungsweise 28 Tage bei den mRNA-Impfstoffen und vier bis sechs Wochen beim Vektorimpfstoff von AstraZeneca. Nur beim Impfstoff von Johnson \& Johnson ist eine einmalige Gabe vorgesehen. „Aktuell werden beide Teilimpfungen der ersten Impfserie mit dem gleichen Impfstoff durchgeführt", erläutert Dr. Barbara Tucek, Leiterin der Abteilung
Klinische Begutachtung Sicherheit \& Wirksamkeit der AGES.

Laut Zwiauer ist aus medizinischer Sicht bei Genesenen eine Impfung ausreichend. Entsprechend der Zulassung der Impfstoffe und aus immunologischer Sicht spricht nichts gegen eine zweimalige Impfung, wenngleich mit einer erhöhten Rate an Impfreaktionen zu rechnen ist. Für den österreichischen grünen Pass reicht eine Impfung völlig aus, international sind jedoch oft auch für diese Gruppe zwei Teilimpfungen vorgeschrieben.

\section{Impfung für Kinder und Jugendliche}

Mittlerweile kann man in Österreich ab dem Alter von 12 Jahren geimpft werden. Kinder und Jugendliche sind allerdings derzeit in der Priorität nachgereiht. „Es gibt jedoch keinen Grund, dieser Altersgruppe die Impfung vorzuenthalten", betont Zwiauer, der auch als Kinderarzt tätig ist. „Kinder können schwer an COVID-19 erkranken. In den letzten Monaten haben wir in Österreich pro Woche ein bis vier Kinder mit COVID-19 auf der Intensivstation behandeln müssen. Bei Krankheiten, bei denen Kinder viel seltener schwer erkranken, sei die Impfung ganz klar außer Streit gestellt. Dann sollten wir das bei COVID-19, das mit einer viel schwereren Krankheitslast einhergeht, erst recht tun."

\section{Forschung geht weiter}

Die EU trifft mittlerweile Vorbereitungen für die Bekämpfung neuer Virusvarianten. Der HERA-Inkubator, 
den die Europäische Kommission im Februar ins Leben gerufen hat, soll dabei helfen. Bogensberger erläutert das Grundprinzip:,"Der HERA-Inkubator bringt Wissenschaft, Wirtschaft und öffentliche Einrichtungen zusammen und soll alle verfügbaren Ressourcen mobilisieren, damit Europa dieser Gefahr frühzeitig entgegentreten kann."

Die Einrichtung des HERA-Inkubators verfolgt gleich mehrere Ziele: Virusvarianten und deren Analyse beziehungsweise eine eventuelle Anpassung der Impfstoffe beschleunigen, die Zulassungsverfahren verkürzen, helfen neue oder umfunktionierte Herstellungsstrukturen zu zertifizieren und die Massenproduktion von angepassten oder neuen COVID-19-Impfstoffen fördern.

\section{Varianten werden genau \\ beobachtet}

Auch die Impfstoffindustrie beobachtet die Varianten genau. „Wir sind gut vorbereitet und können rasch reagieren, falls Anpassungen an neue Virusstämme erforderlich sein sollten", betont Gallo-Daniel. „Bis dato ist das noch nicht nötig." Etwas unklar sei aktuell allerdings noch der Prozess, wie entschieden werde, wann ein speziell gegen bestimmte Varianten adaptierter Impfstoff benötigt wird. Beziehungsweise auch, wann ein Booster mit dem bisherigen Impfstoff benötigt werde.

Von der Zulassungsseite her ist jedenfalls klar: „Laut EMA-Guidance sind für eine potenzielle Auffrischungsimpfung mit einem bereits zugelassenen beziehungsweise an Varianten angepassten Impfstoff keine großen Wirksamkeitsstudien mehr erforderlich", erläutert Tucek. Eingereicht werden müssten Immunogenitätsdaten, die zeigen, dass die freiwilligen Probanden auf den angepassten Impfstoff genauso gut ansprechen wie auf den bisherigen.

Die Impfstoffhersteller sind jedenfalls bereit zu handeln, so es notwendig ist: „Die pharmazeutische Industrie ist weltweit, auf EU-Ebene und auch hier in Österreich in engem Austausch mit den Behörden. Eine enge Abstimmung und Zusammenarbeit ist wichtig, um von Industrieseite zeitnah und effektiv reagieren zu können", fasst Gallo-Daniel zusammen.

\section{Weitere Informationen:}

Österreichischer Verband der Impfstoffhersteller www.oevih.at

Hinweis des Verlags. Der Verlag bleibt in Hinblick auf geografische Zuordnungen und Gebietsbezeichnungen in veröffentlichten Karten und Institutsadressen neutral.

Paediatr. Paedolog. $2021 \cdot 56: 246-247$ https://doi.org/10.1007/s00608021-00929-y

(c) Springer-Verlag GmbH Austria, ein Teil von Springer Nature 2021
Hier steht eine Anzeige. Springer 